\title{
Dual Vascular Endothelial Growth Factor Receptor and Fibroblast Growth Factor Receptor Inhibition Elicits Antitumor Immunity and Enhances Programmed Cell Death-1 Checkpoint Blockade in Hepatocellular Carcinoma
}

\author{
Haijing Deng ${ }^{a, b}$ Anna Kan ${ }^{a, c}$ Ning Lyu ${ }^{a, b}$ Luwen Mud Yi Han ${ }^{a}$ \\ Longzhong Liu ${ }^{a}$ e $Y^{2}$ anyu Zhang ${ }^{a}$ Youfa Duan $^{a}$ Shuangye Liao ${ }^{a}$ \\ Shaolong $\mathrm{Li}^{\mathrm{a}}$ Qiankun Xie ${ }^{\mathrm{a}, \mathrm{f}}$ Tianxiao Gao ${ }^{\mathrm{a}, \mathrm{g}}$ Yanrong $\mathrm{Li}^{\mathrm{a}, \mathrm{b}}$ \\ Zhenfeng Zhang ${ }^{\text {h }}$ Ming Zhao a, b \\ a State Key Laboratory of Oncology in South China, Collaborative Innovation Center for \\ Cancer Medicine, Sun Yat-sen University Cancer Center, Guangzhou, PR China; ${ }^{\mathrm{b}}$ Division \\ of Minimally Invasive Interventional, Sun Yat-sen University Cancer Center, Guangzhou, \\ PR China; ' Department of Hepatobiliary Oncology, Sun Yat-sen University Cancer Center, \\ Guangzhou, PR China; ${ }^{\mathrm{d}}$ Department of Vascular Interventional Radiology, Third Affiliated

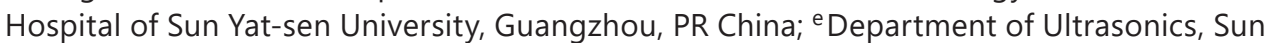 \\ Yat-sen University Cancer Center, Guangzhou, PR China; ${ }^{f}$ VIP Region, Sun Yat-sen University \\ Cancer Center, Guangzhou, PR China; 9 Department of Medical Oncology, Sun Yat-sen \\ University Cancer Center, Guangzhou, PR China; hepartment of Radiology, The Second \\ Affiliated Hospital of Guangzhou Medical University, Guangzhou, PR China
}

\section{Keywords}

Immune checkpoint - Vascular endothelial growth factor A - bFGF · Lenvatinib - Programmed cell death-1 antibodies $\cdot$ Sorafenib · Immunomodulatory $\cdot$ Liver cancer

\begin{abstract}
Background and Aims: Combining anti-angiogenic therapy with immune checkpoint blockade with anti-programmed cell death-1 (PD-1) antibodies is a promising treatment for hepatocellular carcinoma (HCC). Tyrosine kinase inhibitors are well-known anti-angiogenic agents and offer potential for combination with anti-PD-1 antibodies. This study investigated the
\end{abstract}

H.D., A.K., and N.L. contributed equally to this work. 
possible underlying immunomodulatory mechanisms of combined therapy. Methods: HCC tissue samples for RNA-sequencing (RNA-seq) were obtained from patients with differential prognoses following anti-PD-1 treatment. Recombinant basic fibroblast growth factor (bFGF) and vascular endothelial growth factor A (VEGFA) were used to stimulate T cells following lenvatinib or sorafenib treatment, respectively. T cell function was analyzed by flow cytometry and lactate dehydrogenase assay. In vivo experiments were conducted in murine $\mathrm{H} 22$ and Hepa 1-6 competent models of HCC. Local immune infiltration in the tumor microenvironment (TME) was assessed using multicolor flow cytometry. Gene regulation was evaluated by RNA-seq. Microvascular density was measured by immunohistochemistry, and PD-1 ligand (PD-L1) induction was quantified by western blot. Results: The baseline expression of VEGF and fibroblast growth factor (FGF) in patients with progressive disease was significantly higher than in patients achieving stable disease following anti-PD-1 treatment. VEGFA and bFGF significantly upregulated the expression of PD-1, cytotoxic T-lymphocyte-associated protein-4, and Tim-3 on T cells, while inhibiting the secretion of interferon gamma (IFNG) and granzyme B and suppressing $T$ cell cytotoxicity. This immunosuppressive effect was reverted by lenvatinib but not sorafenib. Furthermore, dual lenvatinib/anti-PD-1 antibody therapy led to better antitumor effects than either sorafenib or fibroblast growth factor receptor (FGFR) inhibitor (BGJ398) in H22 murine models of HCC. Combined lenvatinib/anti-PD-1 treatment also led to long-term immune memory formation, while synergistically modulating the TME and enhancing the cytotoxic effect of T cells. Finally, lenvatinib inhibited PD-L1 expression on human umbilical vein endothelial cells, which improved the function of T cells. Conclusions: Inhibition of vascular endothelial growth factor receptor and FGFR augmented the efficacy of anti-PD-1 antibodies. Combined lenvatinib/anti-PD-1 treatment appears to exert antitumor activity by synergistically modulating effector T cell function in the TME and by mutually regulating tumor vessel normalization.

(C) 2020 The Author(s)

Published by S. Karger AG, Basel

\section{Introduction}

Monoclonal antibodies targeting immune checkpoints, either cytotoxic T-lymphocyteassociated protein 4 (CTLA-4) or the programmed cell death-1 (PD-1)/PD-1 ligand (PD-L1) axis, have been a revolutionary development in cancer treatment and are approved for use in a number of malignancies [1-3]. These therapies are being investigated for the treatment of hepatocellular carcinoma (HCC). However, around two-thirds of patients fail to respond to immunotherapy alone $[3,4]$. This highlights the complex interplay of multiple immunosuppressive mechanisms in the tumor microenvironment (TME) and underlines the requirement for the development of more optimal treatment strategies, including combination therapy [5-7].

Vascular normalization has been investigated as a treatment strategy to modulate the immunosuppressive TME and improve responses to immune checkpoint therapy [8-11]. Tyrosine kinase inhibitors (TKI) targeting the vascular endothelial growth factor A/vascular endothelial growth factor receptor (VEGFA-VEGFR) axis have become the preferred treatment for unresectable HCC patients and have been shown to lead to a significant therapeutic effect in phase 3 trials and in clinical practice [12]. TKIs targeting the VEGFA-VEGFR axis also promote vascular normalization. Therefore, the combination of immunotherapy with such TKIs may be an effective combination for the treatment of unresectable HCC [13-16]. In this regard, combined therapy with lenvatinib plus anti-PD-1 antibodies has been designated by the FDA as a breakthrough therapy [17-19]. 
Deng et al.: Dual VEGFR and FGFR Inhibition Elicits Antitumor Immunity and Enhances PD-1 Checkpoint Blockade in HCC

There are many different vascular-targeted TKIs, and each has their own targeting profile. For example, lenvatinib targets fibroblast growth factor receptor 1 (FGFR1) and cabozantinib targets the c-met pathway $[12,20]$. Although the effectiveness of each TKI is supported by abundant clinical investigations as a single drug, the benefit of combining them with immunotherapy remains to be explored. Bevacizumab, for instance, has no significant effect when used alone, while combining bevacizumab with anti-PD-L1 antibodies, however, can lead to a high objective response rate (65.0\%) [21]. Currently, the optimal combination of anti-PD-1 antibodies with vascular-targeted TKIs remains unknown.

This study was conducted to investigate the optimal combination of anti-PD- 1 antibodies and TKIs for unresectable HCC patients and the possible mechanisms of action of combined treatment. Baseline tissue samples for RNA sequencing (RNA-seq) were obtained from HCC patients accepting anti-PD-1 treatment. The tissue samples were also retrospectively analyzed for vascular-related factors affecting the therapeutic effect. TKIs to be investigated were selected through in vitro experiments on T cells. The effectiveness of selected therapy combinations was verified by in vivo experiments. Underlying mechanisms were further explored to provide a rationale for combination therapy with PD-1 antibodies and TKIs.

\section{Experimental Procedures}

\section{Cell Lines}

Cell lines (SK-hep-1, HepG2, SMMC-7721, Huh7, and Hep3B; ATCC, Manassas, VA, USA) derived from human HCC were authenticated by short tandem repeat typing technology and were used for in vitro experiments. Human umbilical vein endothelial cells (HUVEC) were kindly donated by the Gao lab (Sun Yat-sen University, China), and T cells or peripheral blood mononuclear cells (PBMC) were isolated from the blood of patients with HCC treated at the Sun Yat-sen University Cancer Center and healthy donors. The murine HCC cell lines H22 and Hepa 1-6 were used for in vivo experiments.

HCC cells and Hepa 1-6 were cultured in Dulbecco's Modified Eagle's Medium (Gibco, China), and T cells, H22 cells, and PBMCs were cultured in RPMI-1640 (Gibco, China), while HUVEC were cultured in Endothelial Cell Medium (ScienCell, USA). Both mediums were supplemented with $10 \%$ fetal bovine serum (Hyclone, New Zealand) and $1 \%$ penicillin-streptomycin (Gibco, China). All cells were cultured at $37^{\circ} \mathrm{C}$ and $5 \% \mathrm{CO}_{2}$.

\section{Patients and Specimens}

Detailed information for the patients with HCC treated at Sun Yat-sen University Cancer Center involved in the study is listed in the online supplementary Table 1-3 (for all online suppl. material, see www.karger.com/doi/10.1159/000505695). The peripheral blood of patients was collected for PBMC analysis. Following red blood cell lysis, PBMCs were collected by Ficoll gradient separation, after which the "white layer" was cultured in 96-well plates that had been covered with anti-CD3 $(5 \mathrm{ng} / \mathrm{mL})$ and anti-CD28 $(2.5 \mathrm{ng} / \mathrm{mL})$ for stimulation, until stimulated $\mathrm{T}$ cells formed clusters. Written informed consent was obtained from all patients who provided blood samples, and the principles of the Declaration of Helsinki were followed (GZR2019-234).

\section{Cytotoxicity Lactate Dehydrogenase Assay}

The cytotoxicity of T cells was evaluated through lactate dehydrogenase (LDH) level, detected using the Viability/Cytotoxicity Multiplex Assay Kit (CK17, Dojindo, Japan). T cells from HCC patients who had not received targeted treatment were co-cultured with tumor cells treated with VEGFA (50 ng/mL), lenvatinib (120 nM), or sorafenib (120 nM), and the 
Deng et al.: Dual VEGFR and FGFR Inhibition Elicits Antitumor Immunity and Enhances PD-1 Checkpoint Blockade in HCC

supernatant was collected. The LDH levels in different proportions of T cells versus tumor cells were used as a control curve. The experiment was carried out in accordance with the instructions provided by the assay manufacturer.

Tumor Models, Monitoring, and Re-challenge

$\mathrm{Bal} / \mathrm{bc}$ mice and B6/C57 mice, aged 5-6 weeks, were purchased from Guangdong Medical Animal Center (Guangdong, China). Mice were bred and housed under protocols approved by the Institutional Animal Care and Use Committee (IACUC). All animal procedures were approved and performed in accordance with IACUC guidelines (L102012019004A). All animals received humane care according to the criteria outlined in the "Guide for the Care and Use of Laboratory Animals" prepared by the National Academy of Sciences and published by the National Institute of Health (NIH publication 86-23 revised 1985).

For H22 and Hepa 1-6 allografts, $2 \times 10^{6}$ cells were injected subcutaneously in the left abdomen of female Bal/bc and B6/C57 mice, respectively. The maximal tumor diameters were measured in 2 dimensions to calculate tumor volume using the formula $\left(1 / 2 \mathrm{a}^{*} \mathrm{~b} * \mathrm{~b}\right)$. Tumor dimensions were measured using calipers, beginning 3 days after inoculation. Mice were stratified by tumor size to treatment groups to ensure a similar distribution of tumor sizes at baseline. During treatment, mice were sacrificed if they exceeded ethical standards, if the tumor volume reached $2,500 \mathrm{~mm}^{3}$ or if tumor depth exceeded $20 \mathrm{~mm}$. The measurements from such cases were excluded from the analyses. Any mice that achieved a complete response (CR) were re-injected subcutaneously with $2 \times 10^{6}$ cells following a 50-day washout period and followed as described above. Matched controls for re-inoculated mice were taken from cohorts of the same strain of mice that were used as comparator groups for bodyweight changes during treatment, and these control mice were inoculated with an identical number of cancer cells.

\section{Pharmaceuticals and Management}

Lenvatinib (S1164, S5240), sorafenib (S7397), and BGJ398 (S2183) were purchased from Selleck, and anti-PD-1 antibodies were kindly provided by the Zhou lab (Sun Yat-sen University, China) and purchased from BioXCell (BE0146).

Treatment of mice included daily gavage with $0.5 \%$ methylcellulose (control group) or lenvatinib diluted in $0.5 \%$ methylcellulose by daily oral administration at $10 \mathrm{mg} / \mathrm{kg}$ for 10 days or 21 days (lenvatinib combined with PD-1 antibodies group and lenvatinib group), sorafenib diluted in $0.5 \%$ methylcellulose by daily oral administration at $50 \mathrm{mg} / \mathrm{kg}$ for 10 days (sorafenib group and sorafenib combined with PD-1 antibodies group), and BGJ398 diluted in $0.5 \%$ methylcellulose by daily oral administration at $10 \mathrm{mg} / \mathrm{kg}$ for 10 days (BGJ398 combined with PD-1 antibodies group). Murine anti-PD-1 antibodies purchased from BioXcell were diluted with InVivoPure pH 7.0 Dilution Buffer (IP0070) and administered $10 \mathrm{mg} / \mathrm{kg}$ by intraperitoneal injection every 3 days, for a total of 3 doses (lenvatinib, sorafenib, or BGJ398 combined with PD-1 antibodies groups). PD-1 antibodies provided by the Zhou lab were diluted by phosphate-buffered saline (PBS) and $100 \mu \mathrm{g}$ administered by intraperitoneal injection every 3 days, for a total of 6 doses (anti-PD-1 group) and in combination with lenvatinib (combined group).

\section{Tumor Isolation and Flow Cytometry}

Tumors were harvested 21 days after initiation of therapy, and $60 \%$ of the tumors were digested into single cell suspensions, while $40 \%$ of the tumors were sliced into pathological sections. For the former, the cells were passed through a 70-mm filter (352350, Falcon) after which they were collected by Ficoll gradient separation. The "white layer" was stained with antibodies and tested using a BD LSRFortessa ${ }^{\mathrm{TM}} \mathrm{X}-20$ flow cytometer (BD Biosciences, USA).

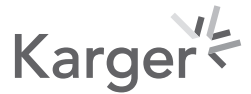


Deng et al.: Dual VEGFR and FGFR Inhibition Elicits Antitumor Immunity and Enhances PD-1 Checkpoint Blockade in HCC

Cell surface staining was performed with CD16-32 antibody in flow cytometry buffer (PBS, supplemented with $2 \%$ fetal bovine serum) according to the manufacturer's instructions. The fluorescent-labeled antibodies used are summarized in online supplementary Table 4. Data were analyzed using FlowJo software (BD Biosciences, USA).

\section{RNA Isolation, Sequencing, and Data Analysis}

Tumor samples were immediately snap-frozen in liquid nitrogen after harvesting. The samples were prepared and RNA-seq was conducted by Novogene (Novogene, China). mRNA was purified from total RNA using poly-T oligo-attached magnetic beads. Fragmentation was carried out using divalent cations under elevated temperature in NEBNext First Strand Synthesis Reaction Buffer ( $5 \times$ ). PCR products were purified (AMPure XP system), and library quality was assessed on the Agilent Bioanalyzer 2100 system. The clustering of the indexcoded samples was performed on a cBot Cluster Generation System using a TruSeq PE Cluster Kit v3-cBot-HS (Illumia, USA) according to the manufacturer's instructions. After cluster generation, the library preparations were sequenced on an Illumina Hiseq platform, and $125 / 150$ bp paired-end reads were generated. Differential expression analysis was performed using the DESeq2 R package (1.16.1). Gene ontology enrichment analysis was implemented by the clusterProfiler R package. Gene ontology terms with corrected $p$ values $<0.05$ were considered significantly enriched by differentially expressed genes.

\section{Immunohistochemistry}

After sacrifice, $40 \%$ of the tumors were fixed in $10 \%$ paraformaldehyde and embedded in paraffin. Tissue sections were cut at a thickness of $4 \mu \mathrm{m}$. After deparaffinization, rehydration, and washing, the sections were blocked with $3 \% \mathrm{H}_{2} \mathrm{O}_{2}$ for $10 \mathrm{~min}$, and microwave antigen retrieval was performed in TrisEDTA (pH 9.0). The sections were then blocked with $5 \%$ bovine serum albumin for 30 min and incubated with primary antibodies and indicated secondary antibodies. Sections were then visualized with DAB. The observation and image acquisition of sections was performed using an advanced research microscope (ECLIPSE 80i NIKON, Japan).

\section{Western Blot and Enzyme-Linked Immunosorbent Assay}

Cells were washed twice with ice-cold PBS and then lysed in lysis buffer with added protease and phosphatase inhibitors for $5 \mathrm{~min}$. Following this, the lysis was boiled for $15 \mathrm{~min}$ at $100^{\circ} \mathrm{C}$. The protein concentration was quantified using the BCA protein assay kit (\#P0012S, Beyotime, China). The primary antibodies used are summarized in online supplementary Table 5. Images were prepared using ChemiDoc Touch (BioRad, China) and analyzed with Image Lab software (BioRad, China).

The level of VEGFA in cell culture supernatants was measured using the Human VEGFA enzyme-linked immunosorbent assay kit (DKW12-1734-096, Dakewe) according to the manufacturer's protocols.

\section{Proteome Profiler Mice XL Cytokine Array}

A membrane-based antibody array (ARY028) for the parallel determination of the relative levels of selected mice cytokines and chemokines was used to validate for analyte detection in serum. Approximately $50 \mu \mathrm{L}$ of peripheral plasma was collected from mice by eyeball extirpating, and the testing was conducted according to the manufacturer's protocol. The levels of a panel of 111 different mice cytokines were analyzed by ImageJ.

\section{Statistical Analysis}

Flow and imaging data were analyzed using FlowJo version 10.4. All statistical analyses and pictures were processed using Prism version 7 (GraphPad, USA). A 2-tailed Student $t$ test 

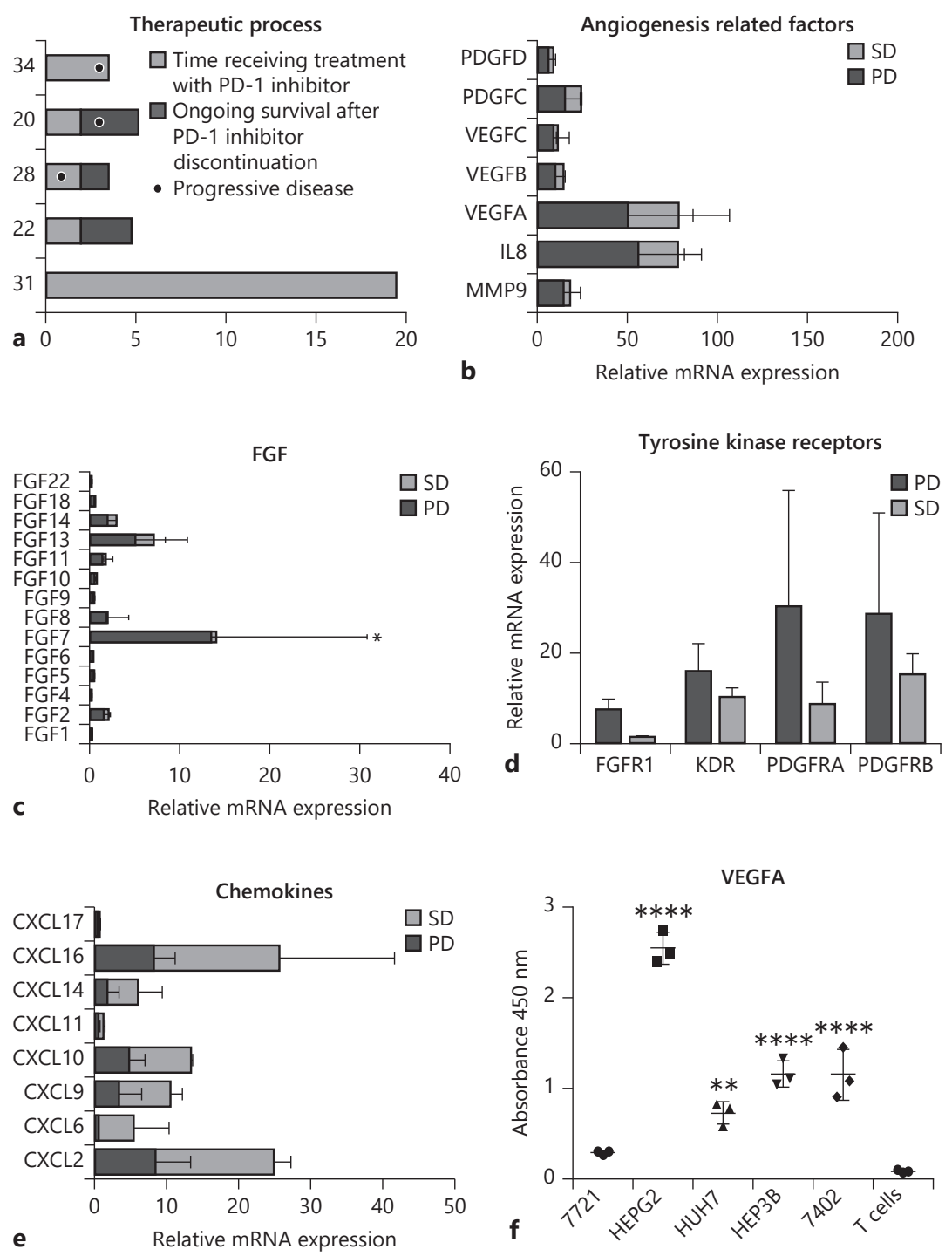

Fig. 1. The expression of angiogenesis-related factors in patients with HCC before initiating anti-PD-1 antibody treatment. Baseline tumor tissue samples from HCC patients treated with PD-1 antibodies were analyzed using RNA-seq. According to treatment response assessed using imaging evaluation (mRECIST), the RNA expression was analyzed (3 patients with partial response [PD] vs. 2 with SD). a Treatment pattern diagram and disease progression for HCC patients. $\mathbf{b}$ Relative mRNA expression of angiogenesis-related factors. c FGF family. Tyrosine kinase receptors (d) and chemokines (e) in patients achieving a PD and SD as tested by RNA-seq. $\mathbf{f}$ The secretion level of VEGFA in HCC cell lines tested by enzyme-linked immunosorbent assay and read by microplate reader at $450 \mathrm{~nm} .{ }^{*} p<0.05,{ }^{* *} p<0.01,{ }^{* * * *} p<0.0001$ (two-way ANOVA). PD-1, programmed cell death-1; VEGFA, vascular endothelial growth factor A; VEGFB, vascular endothelial growth factor B; VEGFC, vascular endothelial growth factor C; FGF, fibroblast growth factor; FGFR1, FGF receptor 1; IL, interleukin; PDGFD, platelet-derived growth factor D; PDGFC, platelet-derived growth factor C; MMP9, matrix metalloprotein; SD, stable disease; PD, progressive disease; TKR, tyrosine kinase receptors; KDR, kinase insert domain receptor; PDGFRA, platelet-derived growth factor receptor A; PDGFRB, platelet-derived growth factor receptor B; CXCL, chemokine (C-X-C motif) ligand. 
and two-way ANOVA were used for comparisons of normally distributed datasets. For survival analysis, $p$ values were computed using the log rank test. $P$ values $<0.05$ were considered significant.

\section{Results}

The VEGF and FGF Family Affect the Efficacy of PD-1 Antibodies by Amplifying the Expression of Angiogenesis-Related Factors in Patients with Progressive Disease

HCC tissue samples were obtained from patients after treatment with anti-PD-1 antibodies for RNA-seq to analyze the relationship between angiogenesis-related factors and therapeutic efficacy. We found that the expression levels of vascular-related factors, such as VEGFA, FGF7, and their receptors VEGFR2 and FGFR1, were generally higher in patients with progressive disease than those with stable disease (Fig. 1a-d). According to analysis using the Kaplan-Meier Plotter database, both high expression of FGF7 (poorly differentiated tumor) and VEGFA were correlated with poor prognosis, although the correlation with FGF7 was not statistically significant (online suppl. Fig. S1A-D). Meanwhile, the chemokine expression profiles for patients with PD and SD were also significantly different, indicating that local microenvironment affects the efficacy of PD-1 therapy (Fig. 1e).

The effect of VEGFA on immune cell infiltration and function has been reported in the past [22]. Aside from VEGFA, our analysis using the TIMER database suggested that FGF7 can also significantly regulate immune infiltration, especially of CD4 T cells (online suppl. Fig. S1E, F). The expression level of VEGFA in human HCC cell lines is high, and previous studies have shown that the expression level of FGF in some HCC cell lines is also high (Fig. 1f) [23, $24]$. Based on the above results, vascular-related factors affect the prognosis of patients treated with immunotherapy, and both VEGFA and FGF7 may affect immune function and the immune composition of the TME, thereby affecting the therapeutic effect.

Lenvatinib Can Reduce the Expression of PD-1, CTLA-4, and Tim-3 on T cells, Promoted by VEGFA and Basic Fibroblast Growth Factor, and Improve the Cytotoxicity of T Cells

Previous studies have reported the effect of VEGFA on the function of CD8 T cells, but few studies have reported the effect of basic fibroblast growth factor (bFGF) and VEGF combined with bFGF on $\mathrm{T}$ cells $[22,25]$. In our study, $\mathrm{T}$ cells were isolated from the peripheral blood of normal donors, HCC patients, and HCC patients treated with anti-PD-1 antibodies. Then, the T cells were stimulated by VEGFA, bFGF, and the combination, respectively, and PD-1 expression was evaluated. We found that T cells from any source had upregulated PD-1 expression after stimulation, and this was particularly noticeable in T

Fig. 2. The effect of stimulation of VEGFA and bFGF on T cells derived from healthy donors and patients with HCC with or without PD-1 antibody treatment were analyzed for PD-1, CTLA-4, and Tim-3 expression and IFNG or GZMB secretion. a Representative images and histogram showing the expression of PD-1 on T cells stimulated by VEGFA ( $50 \mathrm{ng} / \mathrm{mL}$ ), bFGF ( $50 \mathrm{ng} / \mathrm{mL}$ ), or the combination of both. $\mathbf{b}$ Healthy donor-derived T cells were separated and RNA expression of bFGF (FGF2) was analyzed in remaining $\mathrm{T}$ cells and activated T cells by CD3 antibody ( $5 \mathrm{ng} / \mathrm{mL})$ and CD28 antibody $(2.5 \mathrm{ng} / \mathrm{mL})$ and activated T cells treated with lenvatinib (100 nM), sorafenib (100 nM), and BGJ398 (100 nM). c Healthy donor-derived T cells were cultured with HepG2 supernatant and then stimulated by bFGF with gradient concentrations $(0,20,50,100 \mathrm{ng} / \mathrm{mL})$. d Representative images showing CTLA-4 and Tim-3 expression on PD-1+/- T cells. $* p<0.05, * * p<0.01, * * * p<$ 0.001 (two-way ANOVA). VEGF, vascular endothelial growth factor; FGF2, fibroblast growth factor 2; PD-1, programmed cell death-1; HCC, hepatocellular carcinoma; LEN, lenvatinib; SOR, Sorafenib; CD8, cluster of differentiation 8; CTLA4, cytotoxic lymphocyte antigen 4; FSC-H, forward scatter height.

(For figure see next page.) 
Deng et al.: Dual VEGFR and FGFR Inhibition Elicits Antitumor Immunity and Enhances PD-1 Checkpoint Blockade in HCC

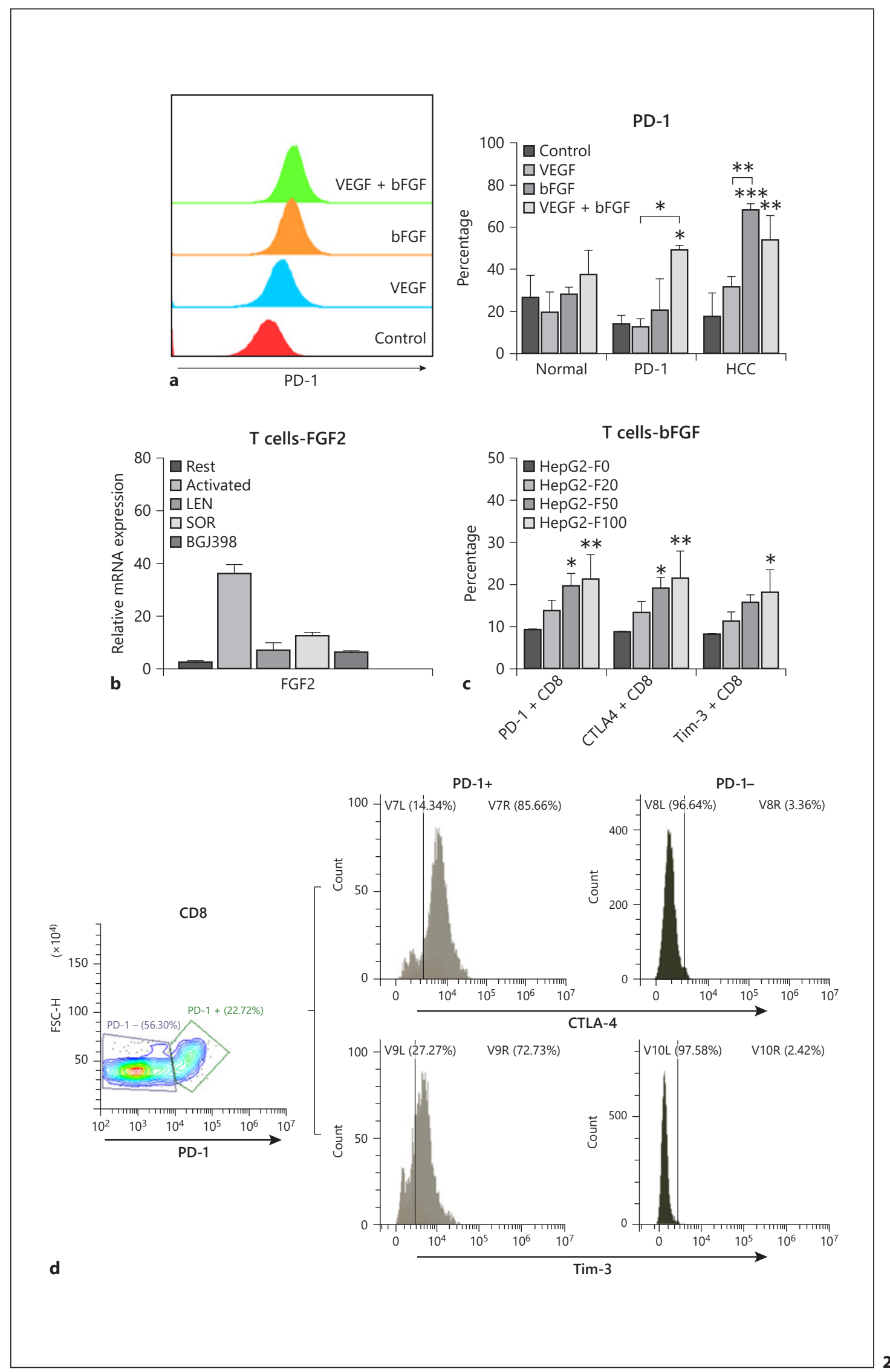


cells derived from HCC patients (Fig. 2a). We then used healthy-donor-derived T cells to activate and detect the expression levels of VEGFA and bFGF. We found that their expression level was significantly upregulated (Fig. $2 \mathrm{~b}$ and online suppl. Fig. S2A). At the same time, we cultured \#T cells with HepG2 supernatant and stimulated them with a concentration gradient of bFGF and then detected the expression of PD-1, CTLA-4, and Tim-3. We found that both checkpoints were significantly upregulated after $72 \mathrm{~h}$ of bFGF stimulation, and this effect was dose-dependent (Fig. 2c). In addition, we also found that T cells with high expression of PD-1 often also highly express CTLA-4 and Tim-3 at the same time (Fig. 2d). We performed an additional T cell activation experiment based on CD3 antibodies (10 ng/ $\mathrm{mL}$ ) to observe the expression of immune checkpoints and used different concentrations of CD3 antibodies as the positive control. We found that VEGFA and bFGF upregulated PD-1 expression, but CTLA-4 expression levels did not change significantly (online suppl. Fig. S2B-E).

In a further experiment, HCC-patient-derived T cells were stimulated by VEGFA and bFGF and simultaneously treated with an identical concentration of lenvatinib (VEGFRs-FGFRstargeting) or sorafenib (VEGFRs-targeting). We found that the expression of PD-1 on T cells was significantly increased after combined stimulation with VEGFA and bFGF. The process could be reverted by treatment with lenvatinib, while PD-1 expression was not downregulated after treatment with sorafenib (Fig. 3a). In a concentration gradient test, lenvatinib at $100 \mathrm{~nm}$ significantly inhibited the expression of PD-1, CTLA4, and Tim-3 on T cells (online suppl. Fig. S2F-H).

We further investigated the effect of lenvatinib on the survival and proliferation of T cells and tumor cells. Lenvatinib of different concentration gradients showed no statistically significant ability to inhibit $\mathrm{T}$ cell proliferation in vitro as well as on tumor cells, as measured by CCK8 (Fig. 3b, online suppl. Fig. S3A-D). Furthermore, an LDH assay was performed to assess $\mathrm{T}$ cell killing function in the co-culture system. The results suggested that $\mathrm{T}$ cell killing function was promoted by lenvatinib but not sorafenib (Fig. 3c). We subsequently measured the secretion of interferon gamma (IFNG) and granzyme B (GZMB) in T cells (without CD3/28 stimulation) in different treatment groups and found that combined stimulation of VEGFA and $\mathrm{bFGF}$ inhibited the secretion of $\mathrm{T}$ cell functional factors, while lenvatinib reversed the process, and sorafenib had no such effect on the secretion of GZMB (Fig. 3d, e). Together, these results suggest that combined stimulation with VEGFA and bFGF can promote the expression of PD-1, CTLA-4, and Tim-3 on T cells, inhibit the function and cytotoxicity of T cells, and that

Fig. 3. The effect of dual targeting of VEGFR and FGFR by lenvatinib on T cell function. HCC-patient-derived $\mathrm{T}$ cells were used to assess the effect of targeting VEGFR and FGFR on T cell function and cytotoxicity. a Representative images showing the expression of PD- 1 on T cells stimulated by VEGFA ( $50 \mathrm{ng} / \mathrm{mL})$ combined with bFGF (50 ng/mL), and then treated with lenvatinib (100 nM) and sorafenib (100 nM), respectively. b The effect of lenvatinib with different concentration gradients $(0,30,60,120,240,480,960,2,000,3,000 \mathrm{~nm})$ on $\mathrm{T}$ cell proliferation, tested by CCK8 and read by microplate reader at $450 \mathrm{~nm}$. c HCC-patient-derived T cells were co-cultured with or without HepG2 cells and treated with or without lenvatinib (100 nM); T cells were cultured with HepG2 cells and treated with Blank, VEGFA $(20 \mathrm{ng} / \mathrm{mL})$, VEGFA (20 ng/mL) + lenvatinib (100 $\mathrm{nM})$, and VEGFA (20 ng/mL) + sorafenib (100 nM). The LDH level was tested and read by microplate reader at $450 \mathrm{~nm}$. Representative images showing the expression of IFNG (d) and GZMB (e) on T cells stimulated by VEGFA or bFGF, respectively, and the combination, and then treated with lenvatinib (100 nM), sorafenib (100 nM), and BGJ398 (FGFRi; $100 \mathrm{nm),} \mathrm{respectively.}{ }^{*} p<0.05$, ${ }^{* *} p<0.01,{ }^{* * *} p<0.001,{ }^{* * * *} p<0.0001$ (two-way ANOVA). VEGF, vascular endothelial growth factor; LEN, lenvatinib; PD-1, programmed cell death-1; LDH, lactate dehydrogenase; IFNG, interferon gamma; GZMB, granzyme B; SSC-A, side scatter; PE-A, K0525, VF, and ECD-A are fluorescent dyes; HepG2 is a hepatoma cell line; BLANK is referred to the control group used in this experiment.

(For figure see next page.)

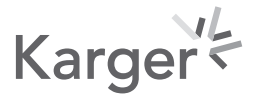




\begin{tabular}{|c|c|c|}
\hline & \multicolumn{2}{|c|}{ Liver Cancer 2020;9:338-357 } \\
\hline Liver Cancer & DOI: $10.1159 / 000505695$ & $\begin{array}{l}\text { (c) } 2020 \text { The Author(s). Published by S. Karger AG, Basel } \\
\text { www.karger.com/lic }\end{array}$ \\
\hline
\end{tabular}

Deng et al.: Dual VEGFR and FGFR Inhibition Elicits Antitumor Immunity and Enhances PD-1 Checkpoint Blockade in HCC

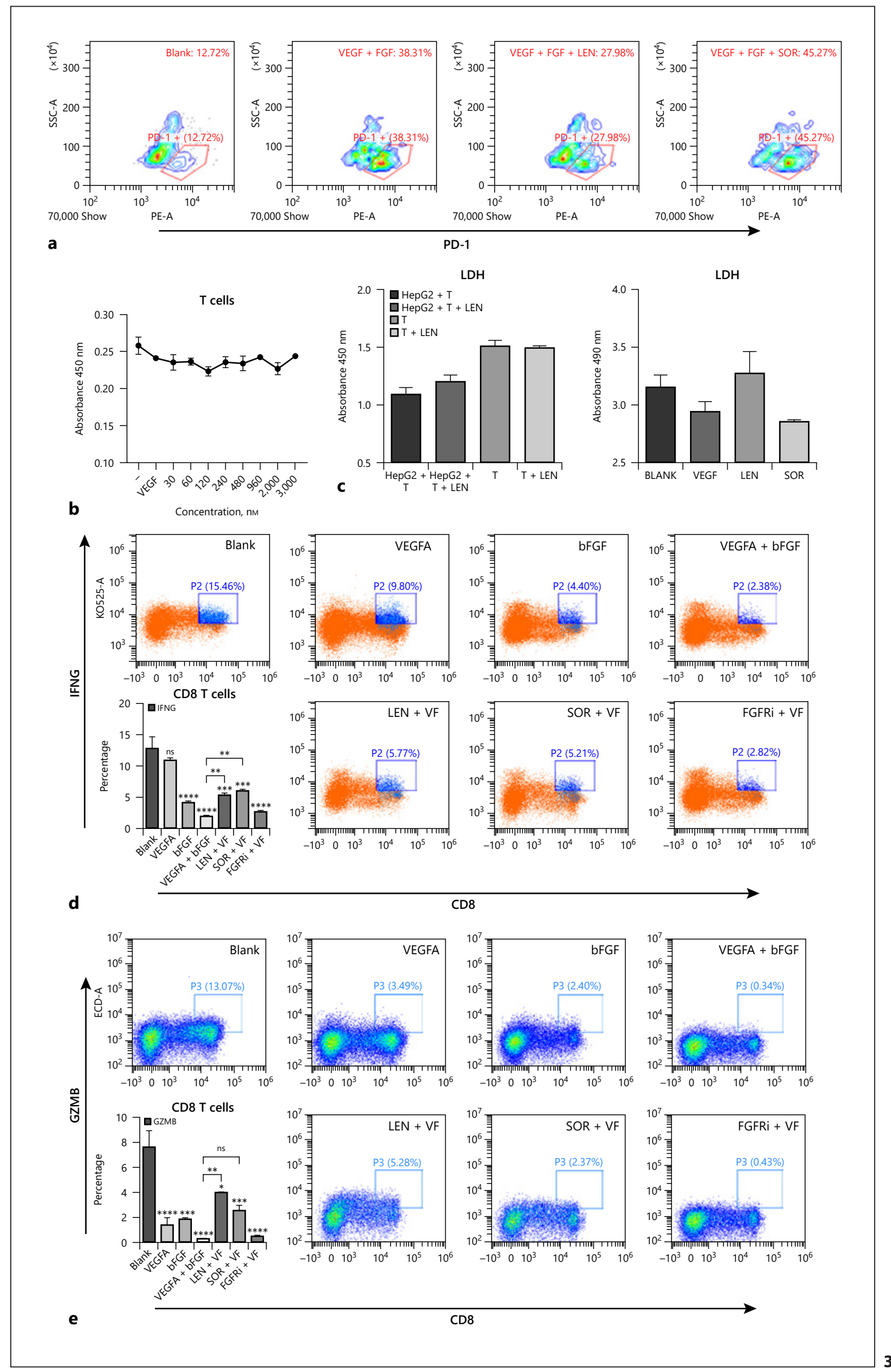


the dual-targeted action of lenvatinib (VEGFR and FGFR) can restore the immune effect of $\mathrm{T}$ cells, but not sorafenib.

In our exploration of mechanisms of action, we found that bFGF can reduce the inhibitory effect of sorafenib on p-ERK in HUVEC, while dual-targeted lenvatinib still played an effective role (online suppl. Fig. S3E). In addition, we detected low VEGFR2 expression on T cells from mouse spleen and intratumoral tissue (online suppl. Fig. S4A). However, we found that the expression of VEGFR2 on the T cells of HCC patients was upregulated compared to those from healthy donors (online suppl. Fig. S4B). Through the treatment of T cells taken from HCC patients with different concentrations of lenvatinib, we found that lenvatinib could reduce the expression of p-PLCr in T cells and that this signal was involved in the upregulation of PD-1 on T cells (online suppl. Fig. S4B, C). Interestingly, we observed that there were 3 populations with different PD-1 expression levels when T cells were activated. We divided them into I, II, and III categories and found that VEGFA promoted PD-1 upregulation in class I and III and bFGF promoted PD-1 upregulation mainly in class II (online suppl. Fig. S5A, B). This may explain why the combination of VEGFA and bFGF significantly upregulated PD-1 expression on T cells.

Combination Treatment with Anti-PD-1 Antibodies and Lenvatinib Has a More Potent Antitumor Effect than Combination with Sorafenib or BGJ398

To directly compare the antitumor effects of different combination treatment strategies, we performed experiments in Bal/bc mice using an $\mathrm{H} 22$ tumor model. After 10 days of administration and observation, we found that both dual targeting of FGFR and VEGFR (lenvatinib) and targeting of VEGFR (sorafenib) or FGFR (BGJ398) alone combined with PD-1 antibodies all had significant antitumor effect, but lenvatinib had a more potent effect than single inhibition of VEGFR or FGFR (Fig. 4a). At the same time, the adverse reactions were milder for combination treatment with lenvatinib compared with mice in the sorafenib combination group, which experienced significant weight loss (Fig. 4b). Lenvatinib combined with PD-1 antibodies significantly inhibited tumor growth, and tumor growth was significantly inhibited in almost all mice in this group. In contrast, in both the sorafenib and the BGJ398 combination groups, there was at least one mouse for which treatment was ineffective (Fig. 4c-g). These results indicate that dual-targeted lenvatinib combined with anti-PD-1 therapy has a more potent antitumor effect in vivo versus combination with sorafenib or BGJ398.

\section{Combined Lenvatinib and Anti-PD-1 Therapy Significantly Inhibits Tumor Growth in vivo} and Induces Long-Term Immune Memory

Combination therapy with lenvatinib and anti-PD-1 antibodies significantly inhibited the growth of murine models of HCC and was more effective than either treatment as monotherapy,

Fig. 4. The effect of lenvatinib, sorafenib, or BGJ398 combined with anti-PD-1 antibodies in vivo. In vivo antitumor experiments in murine models of HCC were performed to compare the anti-tumor effects of lenvatinib, sorafenib, and BGJ398 combined with PD-1 antibodies. a Tumor growth curves over 10 days for the control group ( $n=5$ ), lenvatinib combined with PD-1 antibodies group (10 mg/kg, $200 \mu \mathrm{g} / 3$ days; $n=5$ ), sorafenib combined with PD-1 antibodies group (50 mg/kg, $200 \mu \mathrm{g} / 3$ days; $n=5$ ) and BGJ398 combined with PD-1 antibodies group (10 mg/kg, $200 \mu \mathrm{g} / 3$ days; $n=5$ ) in Balb/C mice models subcutaneously implanted with $\mathrm{H} 22$ cells. b Mean weights for different treatment groups versus control in Balb/C mice models. c Tumor volume changes during treatment in the different groups and the final mean tumor volume at day 10 in the different treatment groups; individual tumor growth curves over the 10 days of treatment in the control group (d); lenvatinib combined with anti-PD-1 antibodies group (e); sorafenib combined with anti-PD-1 antibodies group (f); and BGJ398 combined with anti-PD-1 antibodies group (g). ${ }^{*} p<0.05,{ }^{* *} p<0.01$, **** $p<0.0001$ (one-way/two-way ANOVA). LEN, lenvatinib; PD-1, programmed cell death-1.

(For figure see next page.) 
Deng et al.: Dual VEGFR and FGFR Inhibition Elicits Antitumor Immunity and Enhances PD-1 Checkpoint Blockade in HCC

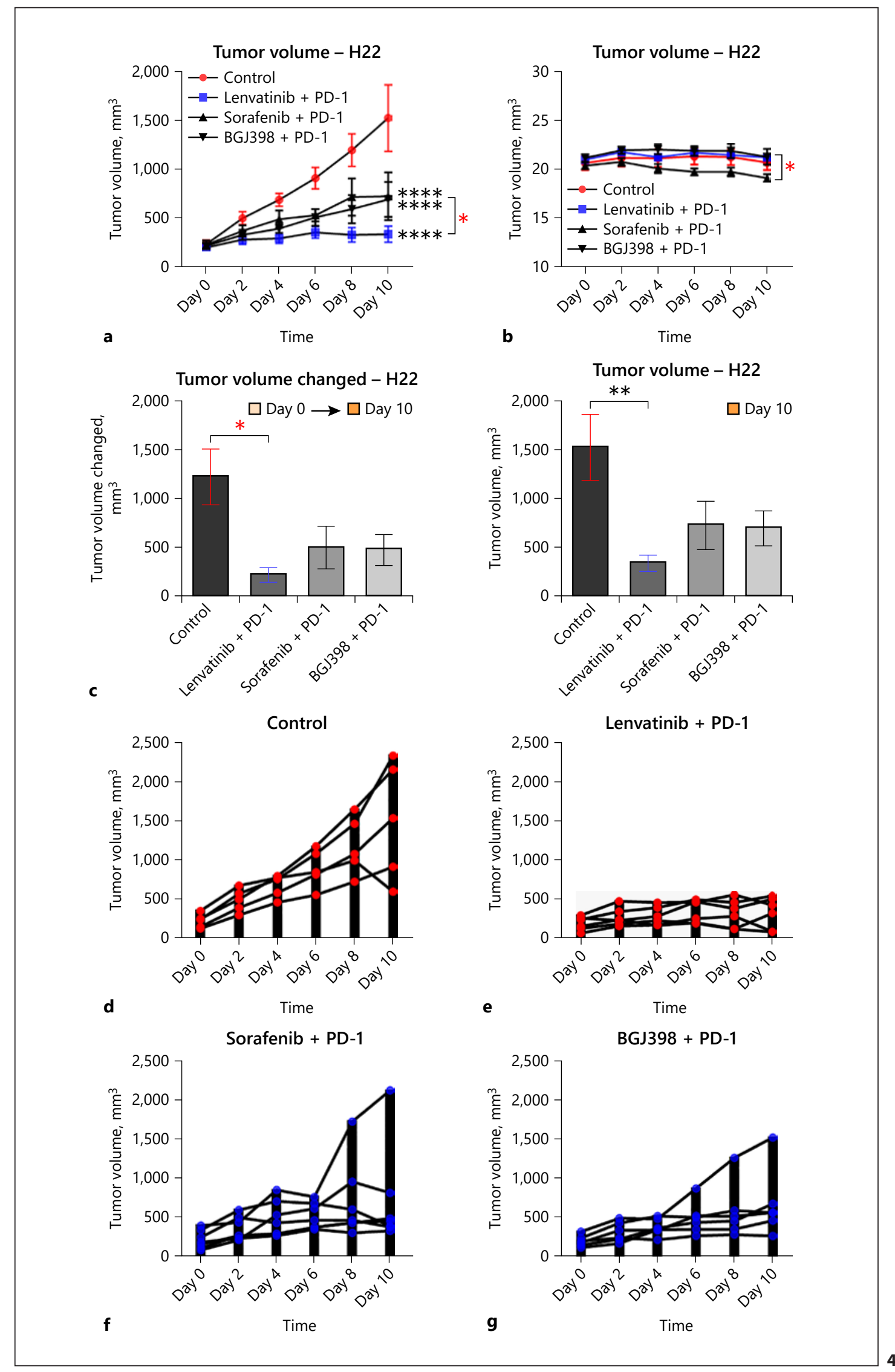




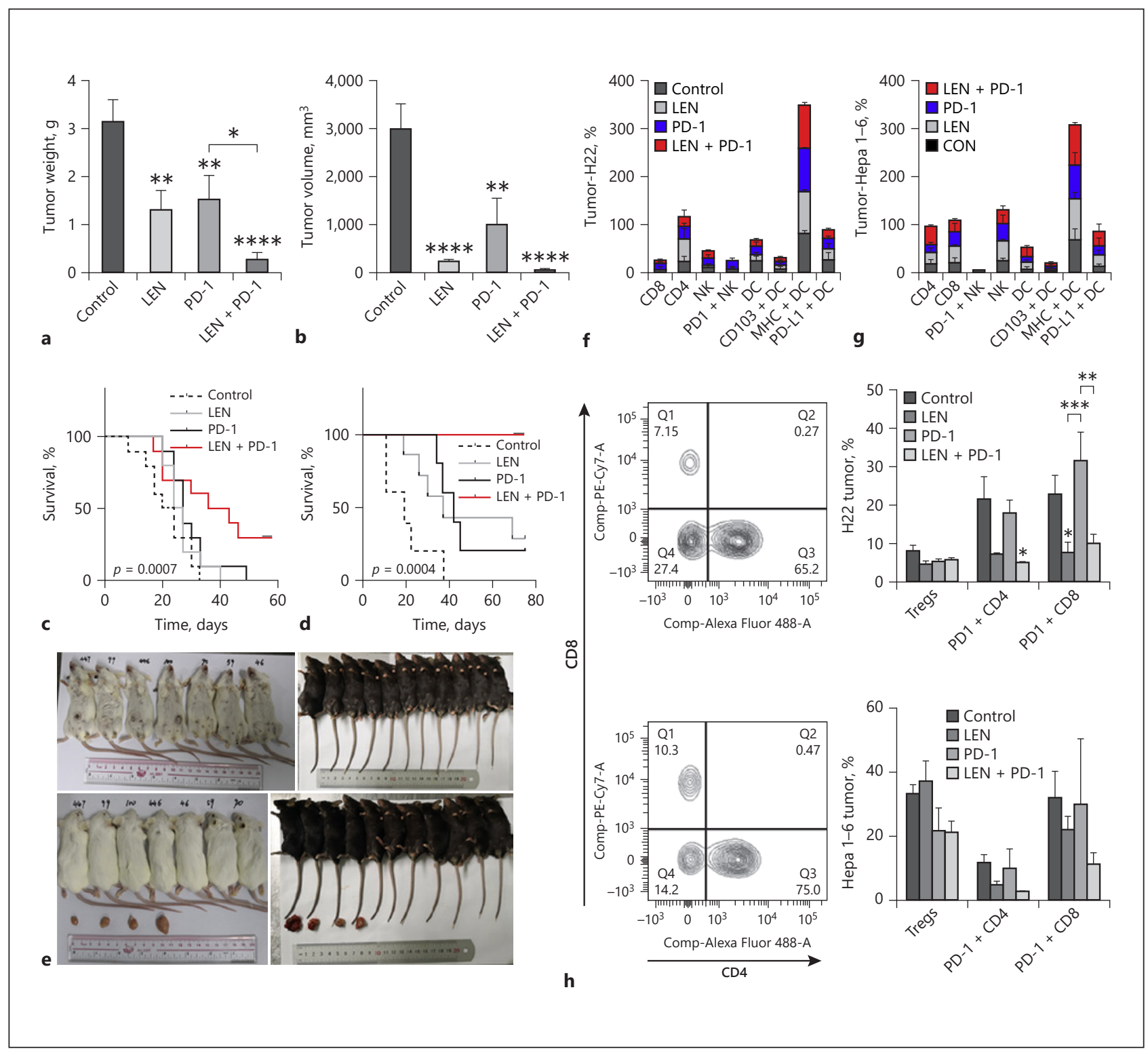

Fig. 5. Antitumor and immunoregulatory effect of combination treatment with lenvatinib and anti-PD-1 antibodies on murine models of HCC. The effect of combination treatment with lenvatinib and anti-PD-1 antibodies was investigated in 2 HCC murine models and the immune TME was tested by flow cytometry. a Mean tumor weights for the lenvatinib group (10 mg/kg), PD-1 antibodies group (100 $\mu \mathrm{g} / 3$ days), and lenvatinib combined with PD-1 antibodies group (10 mg/kg, $100 \mu \mathrm{g} / 3$ days) versus control group treated for 21 days in Balb/C mice models subcutaneously implanted with $\mathrm{H} 22$ cells ( $n=7$ per group). b Mean tumor volume for the different treatment groups versus control over 21 days of treatment in C57BL/ 6 mice models subcutaneously implanted with Hepa 1-6 cells ( $n=5$ per group); survival curve of mice over 24 days of treatment for Balb/C (c) and C57BL/6 (d) mice in the different treatment groups; tumor re-challenge for CR mice in each group after a 50-day washout without treatment in Balb/C mice and C57BL/6 mice versus normal control (e). Histogram showing infiltration of CD4, CD8, NK, and the proportion of PD-1+ NK cells, and infiltration of dendritic cells and the portion of MHC+/PD-L1+ dendritic cells of Balb/C (f) and C57BL/6 mice (g) measured by flow cytometry. $\mathbf{h}$ Representative flow cytometry image showing the change of CD8 $\mathrm{T}$ cells in lenvatinib group versus control group; infiltration of PD-1+CD8, PD-1+ CD4, and T regulatory cell of Balb/C and C57BL/ 6 mice measured by flow cytometry. ${ }^{*} p<0.05,{ }^{* *} p<0.01,{ }^{* * *} p<0.001$, ${ }^{* * * *} p<0.0001$ (two-way ANOVA). LEN, lenvatinib; PD-1, programmed cell death-1; NK, natural killer; MHC+, major histocompatibility complex-positive; PD-L1, PD-1 ligand; DC, dendritic cell; PE-Cy7, a fluorescent dye; CON, control group. 
especially anti-PD-1 antibody monotherapy (Fig. $5 a$, b). In H22 tumors ( $n=7$ per group) combination treatment resulted in a significantly greater reduction in mean tumor weight compared with anti-PD-1 antibody monotherapy. In Hep1-6 tumors ( $n=5$ per group), combination treatment resulted in a significantly reduced mean tumor volume versus control, which was numerically greater than either treatment as monotherapy. The combined therapy significantly prolonged survival in both murine models (Fig. 5c, d). None of the treatment regimens affected the weight of mice or resulted in severe hepatic or renal encephalitis (data not shown).

Combination treatment with lenvatinib and anti-PD-1 antibodies has been shown to lead to formation of immune memory and rejection of re-inoculation of tumor cells into mice that have achieved a CR during treatment [26]. Our results confirmed these findings; mice treated with combination lenvatinib plus anti-PD-1 antibodies that achieved a CR were re-injected with H22 $(n=3)$ or Hepa 1-6 $(n=7)$ tumor cells and compared with matched controls that were inoculated at the same time. Subcutaneous tumors were only successfully propagated in the control animals (Fig. 5e). This suggests that mice achieving a CR after combination therapy had developed immune memory that prevented tumor generation after re-inoculation. Interestingly, the majority of mice that achieved a CR were from the combined treatment group ( $n=3$ in Balb/c with H22; $n=5$ in C57 with Hepa 1-6). These results therefore also suggest that combined therapy with lenvatinib and anti-PD-1 antibodies significantly inhibits tumor growth in vivo and induces long-term immune memory with no obvious adverse effects.

\section{Combined Therapy Has an Extensive Immunomodulatory Effect in the TME, Reduces}

$P D-1+T$ Cells, and Modulates Inflammatory Factors

To explore the mechanisms of combination treatment with lenvatinib and anti-PD-1 antibodies, we investigated the effect of lenvatinib monotherapy and combination therapy on the spleen and the TME of murine models of HCC (Balb/c with H22 and C57 with Hepa 1-6). Combined therapy slightly increased the proportion of natural killer cells and major histocompatibility complex-positive dendritic cells. However, the infiltration of PD-1+ natural killer and dendritic cells had paradoxical results in the 2 murine models, and their variation was slight (Fig. 5f, g and online suppl. Fig. S6). This means that although combined therapy has an extensive immunomodulatory effect in the TME, the investigated immune cells may not be the key mechanism for regulating T cells.

Further investigation of the potential mechanisms regulating $\mathrm{T}$ cells in the TME revealed that combined lenvatinib/anti-PD-1 therapy improved the infiltration of CD4+ and CD8+ T cells, but the result was not statistically significant, and sorafenib had on effect on CD8 infiltration (online suppl. Fig. S7A-C; experimental scheme shown on online suppl. Fig. S7D). In addition, combined therapy with lenvatinib and anti-PD-1 antibodies reduced the infiltration of T regulatory cell and decreased the proportion of PD-1+ CD4+ and CD8+ T cells (Fig. 5h). These results suggest that the effect of combined therapy mainly depends on modulating the function of effector T cells and not by modulating their infiltration. Solid-phase protein microarray analysis of peripheral blood plasma from mice included in the in vivo experiments confirmed that lenvatinib monotherapy and combination therapy could activate the secretion of inflammatory factors, chemokines, and adhesion molecules but decreased factors associated with tumor metastasis (Fig. 6a).

\section{Combined Lenvatinib/Anti-PD-1 Therapy Can Activate the Immune System and Improve the Function of T Cells}

To further investigate the mechanism of combined lenvatinib/anti-PD-1 therapy, we used RNA-seq to compare local genetic modulation. Combined therapy with lenvatinib and anti-PD-1 inhibitors significantly altered gene expression, particularly increasing cluster 4 (immunoglobulin-related genes; Fig. 6b-d, online suppl. Table 6). Pathway enrichment 
analysis showed that combined therapy significantly modulated the gene expression of cytokines mediating signaling pathways compared to PD-1 antibody monotherapy. At the same time, the expression of immune-activation-related markers Cd69, Cd86, Gzmb, Prf1, and IFNG was increased in the combined treatment group (Fig. 6c), especially Gzmb, the cytotoxic factor of CD8 T cells, with combined therapy $(p<0.01)$. Meanwhile, combined therapy extensively changed the expression of chemokines but did not promote macrophage polarization (online suppl. Fig. S7E). What's more, we used multicolor immunohistochemistry of CD8 and PD-1 to verify that combined treatment could reduce PD-1+CD8+ T cells. We found that the co-localization of PD-1 and CD8 was high in the control group but was rare in the combination treatment group (Fig. 6f). These results again demonstrate the activation of the immune system by combined lenvatinib/anti-PD-1 therapy.

\section{Knockdown of VEGFR2 or Targeting by Lenvatinib Can Inhibit IFNG-Induced PD-L1} Expression on HUVEC

Lenvatinib has been reported to exert anti-angiogenic effects both in vitro and in vivo. As shown in Figure 6e, tumor-related angiogenesis was decreased in both murine models following lenvatinib treatment. To further investigate the effect of lenvatinib on PD-L1 expression, we assessed PD-L1 expression in various cells lines by western blot and tested the related pathways in vitro (Fig. 7a, online suppl. Fig. S8A, B). The results showed that when IFNG was stimulated, leading to upregulation of PD-L1 on HUVEC cells, lenvatinib significantly downregulated the expression of PD-L1 and also downregulated the expression of phosphorylated signal transducer and activator of transcription 1 (p-STAT1) (Fig. 7a). This suggests that lenvatinib decreases the expression of PD-L1 on HUVEC by downregulating p-STAT1. This effect may be dependent on VEGFR2 inhibition, shown by downregulation of p-STAT1 and PD-L1 following knockdown of VEGFR2 (Fig. 7b). Furthermore, our results showed that PD-L1 expression on tumor cells was significantly upregulated after IFNG stimulation. The effect of lenvatinib on tumor cells, however, was insignificant (online suppl. Fig. S8A, B). These results show that lenvatinib can decrease PD-L1 expression on HUVEC by inhibiting p-STAT1.

\section{Discussion}

Currently, there are many immunotherapy strategies under investigation, most of which are tentative combination therapies and some of which have a limited mechanistic rationale $[27,28]$. Therefore, immunotherapy has gradually entered the era of combination therapy,

Fig. 6. The effect of combination lenvatinib/anti-PD-1 therapy on the TME. Profiler Mice XL cytokine analysis, RNA-seq, IHC, and TSA-IHC were used to evaluate the effect of lenvatinib combined with PD-1 antibodies therapy on the TME. a Profiler Mice XL cytokine analysis of peripheral blood plasma from tumor-bearing $\mathrm{Balb} / \mathrm{C}$ mice in the different treatment groups. $\mathbf{b}$ Venn diagram analysis of gene expression profiles in tumors in the different treatment groups tested by RNA-seq. c Markers of activated T cells in the different treatment groups tested by RNA-seq. $\mathbf{d}$ Gene expression of Cluster 4 in the different treatment groups tested by RNAseq. e Representative images and histogram of CD31 immunohistochemical staining in the different treatment groups in Balb/C mice and C57BL/6 mice. $\mathbf{f}$ Representative images of CD8 and PD-1 tyramide signal amplification immunohistochemical staining in tumor tissues sampled from tumor-bearing Balb/C mice in the different treatment groups and representative images for PD-1+ CD8 distribution in the combination group versus the control group. ${ }^{*} p<0.05,{ }^{* *} p<0.01,{ }^{* * *} p<0.001,{ }^{* * * *} p<0.0001$ (two-way ANOVA). PD-1, programmed cell death-1; LEN, lenvatinib; ANGPT, angiopoietin 1; TNF-a, tumor necrosis factor-a; ACTB, beta actin, GAPDH, glyceraldehyde-3-phosphate dehydrogenase; Si-NC, the negative control of small interference RNA.

(For figure see next page.)

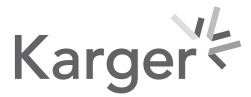




\begin{tabular}{l|l|}
\hline Liver Cancer 2020;9:338-357 \\
\hline DOI: 10.1159/000505695 & $\begin{array}{l}\text { @ 2020 The Author(s). Published by S. Karger AG, Basel } \\
\text { www.karger.com/lic }\end{array}$ \\
\hline
\end{tabular}

Deng et al.: Dual VEGFR and FGFR Inhibition Elicits Antitumor Immunity and Enhances PD-1 Checkpoint Blockade in HCC

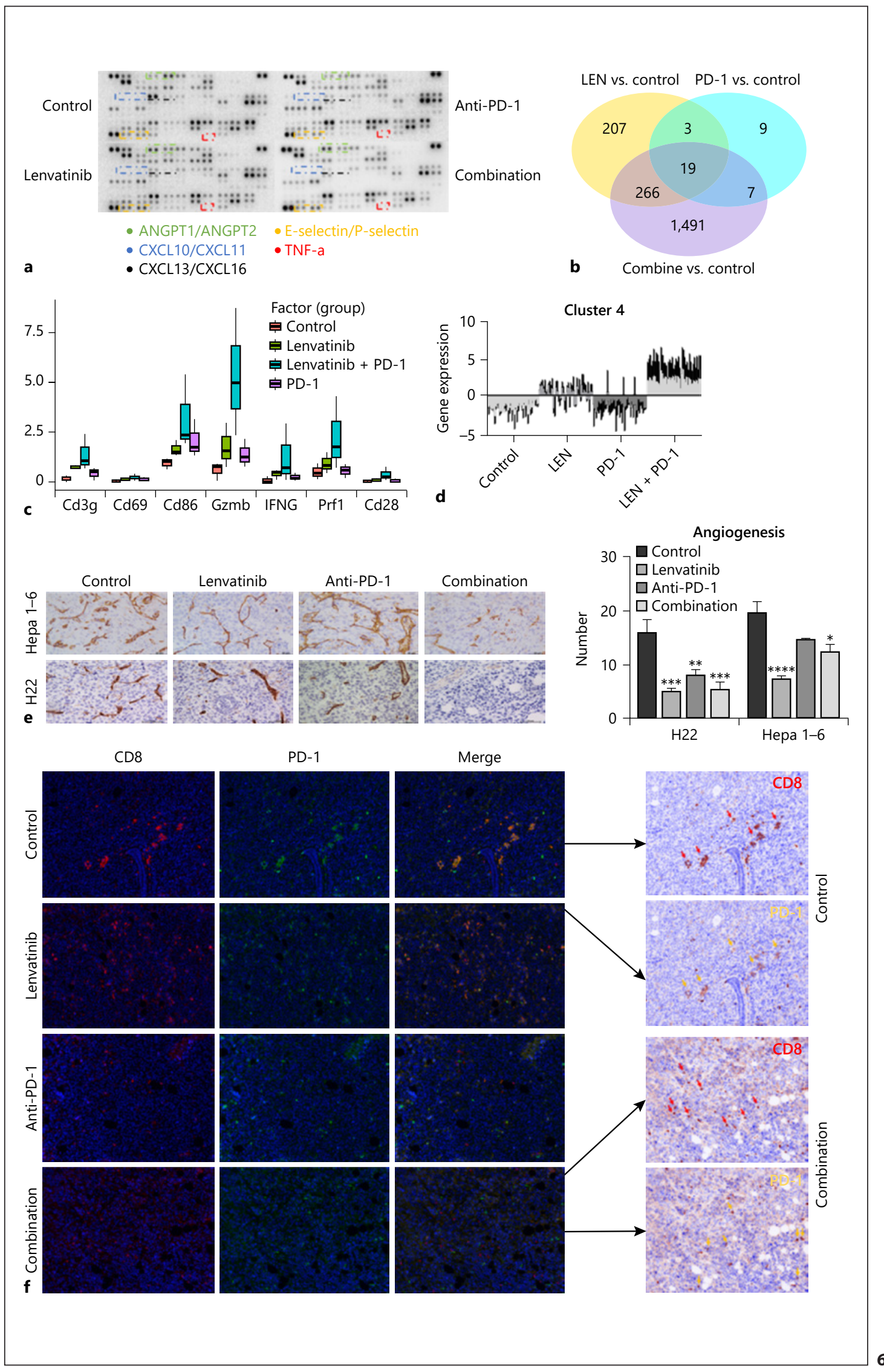

\section{Karger'}


and in-depth investigation of the mechanisms of combined therapy regimens is required. Among these schemes, combining anti-angiogenic therapy with immune checkpoint blockade is a promising approach to fully realize the potential of checkpoint immunotherapy for cancer [29]. Clinically, high overall response rates have been achieved with both bevacizumab $(65.0 \%)$ and lenvatinib (42.3\%) combined with anti-PD-1/PD-L1 antibodies in patients with advanced HCC $[17,30]$. However, there are a number of different anti-angiogenic drugs, and the optimal combination regimen remains to be determined.

In our study, we discovered possible angiogenesis-related factors that affect $\mathrm{T}$ cell function as well as the prognostic factors for PD-1 therapy. Retrospectively, we used RNA-seq to analyze HCC patient tissue samples taken before treatment with anti-PD-1 antibodies. The results showed that the expression of FGF and VEGF family cytokines and their receptors was significantly higher in patients achieving PD compared with those achieving SD. Furthermore, the chemokine expression profiles for PD patients and SD patients were markedly different. This suggests that FGF and VEGF family cytokines as well as the local immune microenvironment may influence the therapeutic effect of PD-1 antibodies. Our study and previous studies also showed that the secretion levels of VEGFA and FGF in HCC cells were higher than normal liver cells, which may affect the immune microenvironment and work to suppress the effectiveness of immunotherapy [22].

We conducted in vitro experiments that verified the stimulation of bFGF and VEGFA can significantly upregulate the expression of PD-1, CTLA-4, and Tim-3 on T cells, inhibit the secretion of IFNG and GZMB in T cells, and reduce the cytotoxicity of T cells. These effects on T cells were even more significant when bFGF and VEGFA were combined. Lenvatinib, however, can reverse this process by simultaneously inhibiting both VEGFR and FGFR. In contrast, sorafenib only targets VEGFR and therefore can only affect the secretion of IFNG in T cells. Nonetheless, it should be noted that lenvatinib has no effect on either the proliferation and survival of T cells or the proliferation, cell cycle, and apoptosis of tumor cells. Previous studies suggested that lenvatinib could inhibit the growth of tumor cells by targeting the autocrine FGF-FGFR pathway [24, 31]. Our results, however, show that lenvatinib has no significant effect on tumor cell growth at a relatively low concentration, but it rather acts as an immunoregulator. In combination, this evidence suggests that lenvatinib may have unique advantages when combined with immunotherapy due to its inhibition of FGFR as well as VEGFR.

In our study, we directly compared lenvatinib with sorafenib (VEGFR inhibitor) and BGJ398 (FGFR inhibitor) in vivo, in mouse models of HCC, and found that lenvatinib had a stronger antitumor effect, higher response rate, and lower incidence of adverse reactions. However, it has previously been found that the dosage of anti-angiogenic therapies has a large effect on local tumor oxygenation status and blood supply, which would therefore inevitably remodel the TME [9]. Therefore, due to the lack of dose investigation in our study, direct comparison of in vivo experiments may show different results at different doses, which still needs further exploration.

In additional in vivo experiments, we found that, compared to sorafenib, lenvatinib is more capable of regulating the infiltration of $\mathrm{T}$ cells. Furthermore, our results also showed that combined treatment with lenvatinib and anti-PD-1 antibodies significantly inhibited tumor growth and prolonged survival in H22 and hepa 1-6 murine models of HCC. It should be mentioned that we extended the observation time and re-inoculated tumor cells after a 51-day washout period for mice achieving a CR during treatment. The results showed that mice from matched control groups could be inoculated successfully, but re-inoculation was rejected in the mice that had achieved a CR. This suggests that long-term immune memory was developed in the mice that achieved a CR during combined treatment with lenvatinib and anti-PD-1 antibodies. We also verified by multicolor flow cytometry, Proteome Profiler Mice XL cytokine analysis, and RNA-seq analysis that the dual-targeting of VEGFR and FGFR reduced the expression of PD- 1 on T cells, improved $\mathrm{T}$ cells function, promoted systematic 


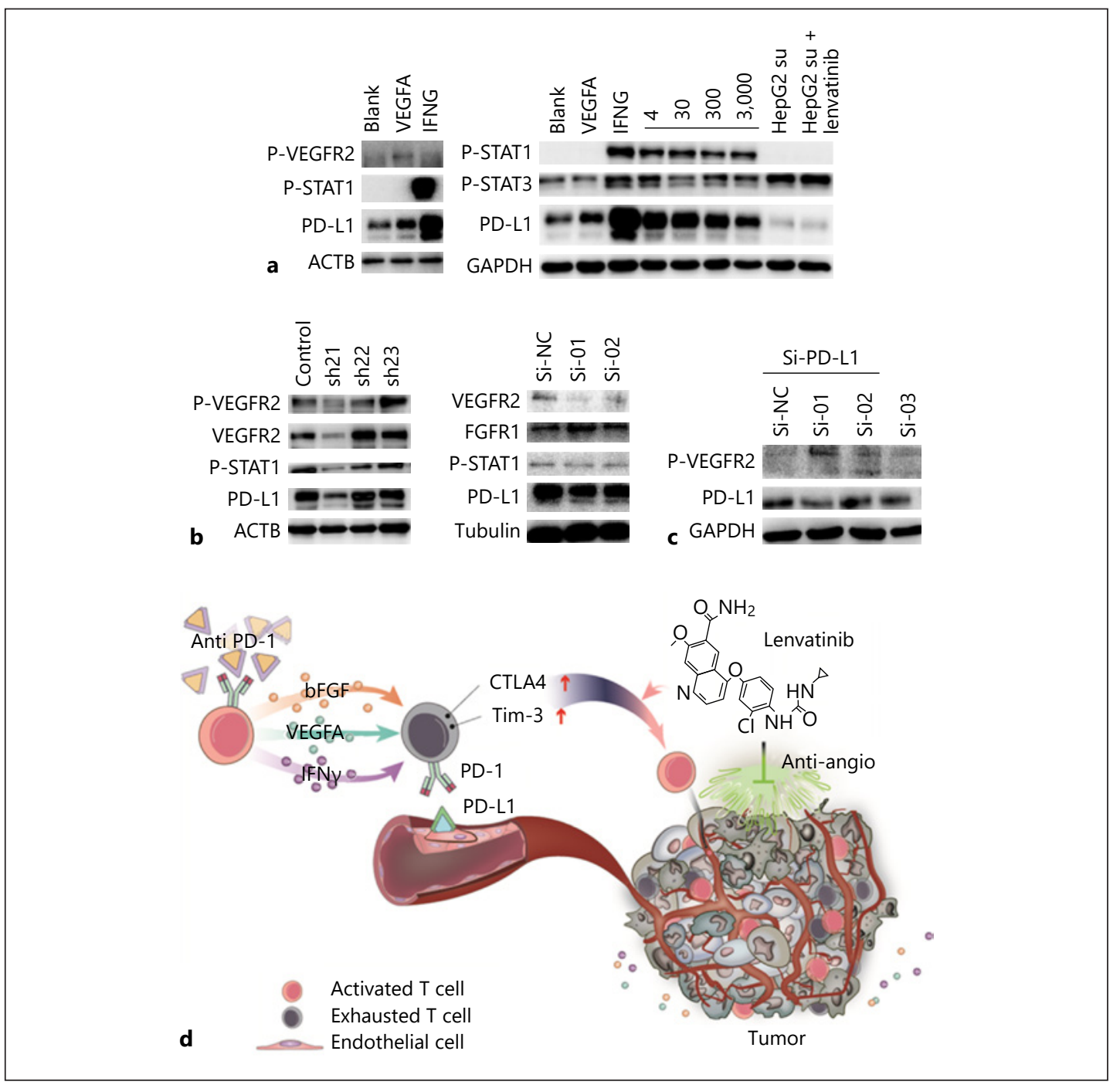

Fig. 7. The effect of lenvatinib on PD-L1 expression on HUVEC. HUVEC were used to test the effect of lenvatinib on PD-L1 expression in vitro. a The effect of VEGFA (20 ng/mL) and lenvatinib with gradient concentration $(4,30,300,3,000 \mathrm{nM})$ on PD-L1 expression following stimulation by IFNG $(10 \mathrm{ng} / \mathrm{mL})$; the effect of HepG2 supernatant on PD-L1 expression in HUVEC. b The effect of downregulating VEGFR2 (sh21, sh22, sh23; si-01, si-02) in HUVEC on p-STAT1 and PD-L1 following stimulation by IFNG $(10 \mathrm{ng} / \mathrm{mL})$. c The effect of downregulating PD-L1 (si-01, si-02, si-03) in HUVEC on p-VEGFR2 following stimulation by VEGFA (50 ng/ $\mathrm{mL}$ ). d Diagram showing that dual targeting VEGFR and FGFR elicits antitumor immunity and enhances PD-1 checkpoint blockade in HCC. VEGF, vascular endothelial growth factor; LEN, lenvatinib; PD-1, programmed cell death-1; IFNG, interferon gamma; PD-L1, PD-1 ligand; HN and NH are both amines

inflammatory cytokines secretion, and changed the chemokine spectrum, thus improving the curative effect of PD-1 antibodies.

Our study also showed that lenvatinib leads to vascular regulation. We measured the density of local tumor microvessels and found that the density was synergistically reduced during combined lenvatinib/anti-PD-1 therapy. At the same time, our in vitro investigation also found that lenvatinib reduced the expression of PD-L1 on HUVEC and further restored the function of T cells without affecting the expression of PD-L1 on tumor cells, thus retaining tumor cells' sensitivity to PD-1 monoclonal antibodies. 
Our study was conducted to investigate the optimal TKI combination with PD-1 antibodies for patients with unresectable HCC. However, we did not explore drug dosage and this is a primary weakness of this study. Our investigation also requires further clinical verification.

In summary, our study revealed the advantages of targeting both VEGFR and FGFR. First, we emphasized the role of bFGF in regulating the function of T cells. Second, we found that it is more conducive to elucidate the underlying mechanism of lenvatinib combined with PD-1 antibodies by including the immune regulation effect of bFGF (Fig. 7d). Our study therefore provides a theoretical basis for the clinical application of combined treatment with lenvatinib and anti-PD-1 antibodies.

\section{Acknowledgments}

Thanks to the selfless help of the Gao lab and Zhou lab, the strong support of the patients in this experiment, and the sacrifice of experimental animals for scientific inquiry. The road ahead will be long, our climb will be steep.

\section{Statement of Ethics}

The peripheral blood of patients with HCC at Sun Yat-sen University Cancer Center was collected for PBMC analysis. Written informed consent was obtained from all patients who provided blood samples and the principles of the Declaration of Helsinki were followed. All animal procedures were approved and performed in accordance with IACUC guidelines (L102012019004A). The datasets supporting the conclusions of this article are available in the Research Data Deposit repository. The RDDN of the dataset for this study is RDDB2019000763.

\section{Funding Sources}

This work was primarily supported by National Natural Science Foundation of China (No. 81771956) and the National Natural Science Foundation of Guangdong (2016A030313282 and 2017A020215149). Editorial support for the writing of this manuscript was paid for by Eisai and provided by Jake Burrell, PhD (Rude Health Consulting).

\section{Disclosure Statement}

The authors have no conflict of interest.

\section{Author Contributions}

H.D., A.K., and N.L. performed the described studies, analyzed data, and prepared the manuscript. L.M., S.L., Q.X., and L.L. provided clinical suggestions. Y.H., Y.D., Y.Z., Y.L., S.L., and T.G. provided materials and assisted in experiments. Z.Z. and M.Z. advised on study design and prepared the manuscript. All authors read and approved the final manuscript.

\section{References}

1 Topalian SL, Hodi FS, Brahmer JR, Gettinger SN, Smith DC, McDermott DF, et al. Safety, activity, and immune correlates of anti-PD-1 antibody in cancer. N Engl J Med. 2012 Jun;366(26):2443-54.

2 Hamid O, Robert C, Daud A, Hodi FS, Hwu WJ, Kefford R, et al. Safety and tumor responses with lambrolizumab (anti-PD-1) in melanoma. N Engl J Med. 2013 Jul;369(2):134-44.

3 El-Khoueiry AB, Sangro B, Yau T, Crocenzi TS, Kudo M, Hsu C, et al. Nivolumab in patients with advanced hepatocellular carcinoma (CheckMate 040): an open-label, non-comparative, phase 1/2 dose escalation and expansion trial. Lancet. 2017 Jun;389(10088):2492-502. 


\begin{tabular}{|c|c|}
\hline Liver Cancer 2020;9:338-357 & \\
\hline DOI: $10.1159 / 000505695$ & $\begin{array}{l}\text { (c) } 2020 \text { The Author(s). Published by S. Karger AG, Basel } \\
\text { www.karger.com/lic }\end{array}$ \\
\hline
\end{tabular}

4 Finkelmeier F, Waidmann 0, Trojan J. Nivolumab for the treatment of hepatocellular carcinoma. Expert Rev Anticancer Ther. 2018 Dec;18(12):1169-75.

5 Wang Q, Wu X. Primary and acquired resistance to PD-1/PD-L1 blockade in cancer treatment. Int Immunopharmacol. 2017 May;46:210-9.

6 Granier C, De Guillebon E, Blanc C, Roussel H, Badoual C, Colin E, et al. Mechanisms of action and rationale for the use of checkpoint inhibitors in cancer. ESMO Open. 2017 Jul;2(2):e000213.

7 Jenkins RW, Barbie DA, Flaherty KT. Mechanisms of resistance to immune checkpoint inhibitors. Br J Cancer. 2018 Jan;118(1):9-16.

8 Cook KM, Figg WD. Angiogenesis inhibitors: current strategies and future prospects. CA Cancer J Clin. 2010 Jul-Aug;60(4):222-43.

9 Huang Y, Yuan J, Righi E, Kamoun WS, Ancukiewicz M, Nezivar J, et al. Vascular normalizing doses of antiangiogenic treatment reprogram the immunosuppressive tumor microenvironment and enhance immunotherapy. Proc Natl Acad Sci USA. 2012 Oct;109(43):17561-6.

10 Tamura R, Tanaka T, Ohara K, Miyake K, Morimoto Y, Yamamoto Y, et al. Persistent restoration to the immunosupportive tumor microenvironment in glioblastoma by bevacizumab. Cancer Sci. 2019 Feb;110(2):499-508.

11 Peng S, Wang Y, Peng H, Chen D, Shen S, Peng B, et al. Autocrine vascular endothelial growth factor signaling promotes cell proliferation and modulates sorafenib treatment efficacy in hepatocellular carcinoma. Hepatology. 2014 Oct;60(4):1264-77.

12 Kudo M. Lenvatinib May Drastically Change the Treatment Landscape of Hepatocellular Carcinoma. Liver Cancer. 2018 Mar; 7(1):1-19.

13 Waidmann 0. Recent developments with immunotherapy for hepatocellular carcinoma. Expert Opin Biol Ther. 2018 Aug;18(8):905-10.

14 Hato T, Zhu AX, Duda DG. Rationally combining anti-VEGF therapy with checkpoint inhibitors in hepatocellular carcinoma. Immunotherapy. 2016;8(3):299-313.

15 Schmittnaegel M, Rigamonti N, Kadioglu E, Cassará A, Wyser Rmili C, Kiialainen A, et al. Dual angiopoietin-2 and VEGFA inhibition elicits antitumor immunity that is enhanced by PD-1 checkpoint blockade. Sci Transl Med. 2017 Apr;9(385):9.

16 Tian L, Goldstein A, Wang H, Ching Lo H, Sun Kim I, Welte T, et al. Mutual regulation of tumour vessel normalization and immunostimulatory reprogramming. Nature. 2017 Apr;544(7649):250-4.

17 Kudo M, Finn RS, Qin S, Han KH, Ikeda K, Piscaglia F, et al. Lenvatinib versus sorafenib in first-line treatment of patients with unresectable hepatocellular carcinoma: a randomised phase 3 non-inferiority trial. Lancet. 2018 Mar;391(10126):1163-73.

18 Grünwald V, Powles T, Choueiri TK, Hutson TE, Porta C, Eto M, et al. Lenvatinib plus everolimus or pembrolizumab versus sunitinib in advanced renal cell carcinoma: study design and rationale. Future Oncol. 2019 Mar; 15(9):929-41.

19 Makker V, Rasco D, Vogelzang NJ, Brose MS, Cohn AL, Mier J, et al. Lenvatinib plus pembrolizumab in patients with advanced endometrial cancer: an interim analysis of a multicentre, open-label, single-arm, phase 2 trial. Lancet Oncol. 2019 May;20(5):711-8.

20 Abou-Alfa GK, Meyer T, Cheng AL, El-Khoueiry AB, Rimassa L, Ryoo BY, et al. Cabozantinib in Patients with Advanced and Progressing Hepatocellular Carcinoma. N Engl J Med. 2018 Jul;379(1):54-63.

21 Kudo M. Systemic Therapy for Hepatocellular Carcinoma: latest Advances. Cancers (Basel). 2018 Oct;10(11): 10.

22 Voron T, Colussi O, Marcheteau E, Pernot S, Nizard M, Pointet AL, et al. VEGF-A modulates expression of inhibitory checkpoints on CD8+ T cells in tumors. J Exp Med. 2015 Feb;212(2):139-48.

23 Tovar V, Cornella H, Moeini A, Vidal S, Hoshida Y, Sia D, et al. Tumour initiating cells and IGF/FGF signalling contribute to sorafenib resistance in hepatocellular carcinoma. Gut. 2017 Mar;66(3):530-40.

24 Yamamoto Y, Matsui J, Matsushima T, Obaishi H, Miyazaki K, Nakamura K, et al. Lenvatinib, an angiogenesis inhibitor targeting VEGFR/FGFR, shows broad antitumor activity in human tumor xenograft models associated with microvessel density and pericyte coverage. Vasc Cell. 2014 Sep;6(1):18.

25 Li P, Huang T, Zou Q, Liu D, Wang Y, Tan X, et al. FGFR2 Promotes Expression of PD-L1 in Colorectal Cancer via the JAK/STAT3 Signaling Pathway. J Immunol. 2019 May;202(10):3065-75.

26 Kato Y, Tabata K, Kimura T, Yachie-Kinoshita A, Ozawa Y, Yamada K, et al. Lenvatinib plus anti-PD-1 antibody combination treatment activates CD8+ T cells through reduction of tumor-associated macrophage and activation of the interferon pathway. PLoS One. 2019 Feb;14(2):e0212513.

27 Wei SC, Levine JH, Cogdill AP, Zhao Y, Anang NAS, Andrews MC, et al. Distinct Cellular Mechanisms Underlie Anti-CTLA-4 and Anti-PD-1 Checkpoint Blockade. Cell 2017;170:1120-33.e17.

28 Sanmamed MF, Chen L. A Paradigm Shift in Cancer Immunotherapy: From Enhancement to Normalization. Cell. 2018 Oct;175(2):313-26.

29 Allen E, Jabouille A, Rivera LB, Lodewijckx I, Missiaen R, Steri V, et al. Combined antiangiogenic and anti-PDL1 therapy stimulates tumor immunity through HEV formation. Sci Transl Med. 2017 Apr;9(385):9.

30 Kudo M. Extremely High Objective Response Rate of Lenvatinib: Its Clinical Relevance and Changing the Treatment Paradigm in Hepatocellular Carcinoma. Liver Cancer. 2018 Sep;7(3):215-24.

31 Hoshi T, Watanabe Miyano S, Watanabe H, Sonobe RM, Seki Y, Ohta E, et al. Lenvatinib induces death of human hepatocellular carcinoma cells harboring an activated FGF signaling pathway through inhibition of FGFRMAPK cascades. Biochem Biophys Res Commun. 2019 May;513(1):1-7. 